\title{
Finite difference modeling of geoacoustic interaction at anelastic seafloors
}

\author{
Ralph A. Stephen and Stephen A. Swift \\ Woods Hole Oceanographic Institution, Woods Hole, Massachusetts 02543
}

(Received 19 February 1993; accepted for publication 13 July 1993)

\begin{abstract}
A major problem in understanding seismic wave propagation in the seafloor is to distinguish between the loss of energy due to intrinsic attenuation and the loss of energy due to scattering from fine scale heterogeneities and bottom roughness. Energy lost to intrinsic attenuation (heat) disappears entirely from the system. Energy lost to scattering is conserved in the system and can appear in observations as incoherent noise (reverberation, time spread, angle spread) and/or mode converted waves. It has been shown by a number of investigators that the seafloor scattering problem can be addressed by finite difference solutions to the elastic wave equation in the time domain. However previous studies have not considered the role of intrinsic attenuation in the scattering process. In this paper, a formulation is presented which includes the effects of intrinsic attenuation in a two-dimensional finite difference formulation of the elastodynamic equations. The code is stable and yields valid attenuation results.
\end{abstract}

PACS numbers: 43.20.Bi, 43.20.Gp, 43.20.Jr, 43.30.Es

\section{INTRODUCTION}

The finite difference synthetic seismogram method has proven to be a useful technique for studying acoustic bottom interaction. It is one of the few techniques which can treat full wave elastic (compressional and shear wave) scattering from both surface and volume heterogeneities with scale lengths on the order of seismic wavelengths (Dougherty and Stephen, 1988, 1991). Since the seafloor is rough and laterally heterogeneous over a broad range of scales, scattering from the seafloor is significant in many areas of ocean acoustics.

The finite difference method provides the capability for studying the physical mechanisms of scattering in complex environments. However, until recently, the time domain finite difference method did not allow for the effects of intrinsic attenuation. Day and Minster (1984) proposed a method for including intrinsic attenuation in a time domain finite difference scheme and gave an example based on one-dimensional wave propagation (waves on a string). We have implemented the approach of Day and Minster in a two-dimensional finite difference scheme. This scheme provides the capability to address the effects of scattering and intrinsic attenuation in the same formulation.

Attenuation is important in bottom interacting ocean acoustics because it impacts the range to which bottom propagating energy is significant and it plays a role in the physical processes of scattering from rough and laterally heterogeneous seafloors. Field measurements of attenuation give effective attenuation which is a combination of both scattering and intrinsic attenuation (Jacobson and Lewis, 1990 for example). For some applications in bottom interacting acoustics the effective attenuation is the primary quantity of interest. It measures the amount of energy lost from the transmission loss curve, or coherent arrival, that cannot be explained by spreading losses and multipathing. In some cases, even conversions to shear waves are treated as an attenuation.

The distinction between intrinsic attenuation and scattering is important. Intrinsic attenuation represents energy lost to the system through internal friction (Jackson and Anderson, 1970). Scattering represents energy lost from a particular coherent path either to another coherent path (as for shear waves) or to incoherent energy. Not much can be done about intrinsic attenuation other than to measure it, but system performance can be improved by understanding and exploiting scattered energy. In the case of multiple coherent paths, processing can be carried out to optimize the total energy received on all paths. In the case of incoherent scattering, there is an increase in the noise floor due to the source generated noise, which yields an upper bound on the signal-to-noise ratio independent of true ambient noise. Different environments scatter energy in different frequency bands and have different, frequencydependent, signal-to-noise ratio ceilings. The modeling capability described here can be used to distinguish the effects of intrinsic attenuation and scattering for realistic seafloor structures.

\section{THE WAVE EQUATION FOR HETEROGENEOUS ANELASTIC MEDIA}

To incorporate attenuation in our two-dimensional finite difference formulation we use the method outlined in Day and Minster (1984). Their approach uses Padé approximants to a general time-dependent stress-strain relation. Stress-strain relations corresponding to a broad range of attenuation mechanisms can be treated.

Previous work on finite difference solutions to the elastic wave equation for isotropic, heterogeneous media addressed the second-order partial differential equation in terms of particle displacement (Stephen, 1988 for example): 


$$
\begin{aligned}
\rho \ddot{\mathbf{u}}= & (\lambda+\mu) \nabla(\nabla \cdot \mathbf{u})+\mu \nabla^{2} \mathbf{u} \\
& +[\nabla \lambda(\nabla \cdot \mathbf{u})+\nabla \mu \times(\nabla \times \mathbf{u})+2(\nabla \mu \cdot \nabla) \mathbf{u}]
\end{aligned}
$$

or equivalently in tensor notation,

$\rho \ddot{u}_{i}=(\lambda+\mu) u_{j, j i}+\mu u_{i, j j}+\lambda_{, i} u_{k, k}+\mu_{, j}\left(u_{i, j}+u_{j, i}\right)$,

where $\mathrm{u}$ is the particle displacement vector, $\lambda$ and $\mu$ are Lamé's parameters, and $\rho$ is the density. For simplicity, we consider here a Cartesian coordinate system.

The initial conditions for (1) are that the particle displacement and particle velocity are zero everywhere. Various body forces and boundary conditions are incorporated with (1) depending on applications. For example, a source inside the finite difference grid can be introduced as a distribution of time-dependent body forces (Alterman and Aboudi, 1970) or a source outside the finite difference grid can be introduced as a time-dependent boundary condition (Stephen, 1983). Also, in order to minimize the size of the computational grid, absorbing boundaries are frequently used to satisfy the Sommerfeld radiation condition (Clayton and Engquist, 1977, for example). The various options for body forces and absorbing boundaries do not affect the treatment of anelasticity and will not be discussed further in this paper.

To implement a time-dependent stress-strain relation for anelasticity it is more convenient to consider the system of equations from which the wave equation (1) was derived. Any code which computes the wave equation directly can easily be reconfigured to compute the wave equation by successively computing the equation of motion (2), the stress-strain relation for isotropic media (generalized Hooke's law) (3), and the definition of infinitesimal strain (4) (Aki and Richards, 1980):

$$
\begin{aligned}
& \rho \ddot{u}_{i}=\tau_{j i, j}, \\
& \tau_{i j}=\lambda \delta_{i j} e_{k k}+2 \mu e_{i j}, \\
& e_{i j} \equiv \frac{1}{2}\left(u_{i, j}+u_{j, i}\right),
\end{aligned}
$$

where $\tau_{i j}$ and $e_{i j}$ are the stress and strain tensors, respectively. Because of symmetry conditions each of the stress and strain tensors have only six independent components (e.g., $\tau_{11}, \tau_{22}, \tau_{33}, \tau_{12}, \tau_{23}$, and $\tau_{13}$ ).

We outline here the key relationships, based on elastic moduli and stress relaxation functions, that are required to introduce anelasticity into our finite difference code. We use the nomenclature of Nowick and Berry (1972) and complete derivations of the equations are given in their book. Other authors often use compliances and creep and elastic aftereffect functions. The relationships between the various functions, moduli, and compliances are given in Nowick and Berry. They also give a review of various physical mechanisms responsible for anelasticity which are beyond the scope of this paper.

Ideal elastic mechanical behavior satisfies, by definition, three postulates (Nowick and Berry, 1972). (i) For every stress there is a unique equilibrium value of strain (and vice versa). A corollary to this postulate is complete recoverability. That is the body will return to its original shape after the applied stress is removed. (ii) The equilibrium response is achieved instantaneously. (iii) The stressstrain relation is linear.

An anelastic material satisfies all these postulates except postulate (ii). The medium continues to deform with time while the force is applied and continues to recover its original shape after the force is removed. Anelasticity also includes the case where there is an instantaneous deformation followed by a time-dependent deformation. A viscoelastic medium does not satisfy postulates (i) and (ii). In addition to having a time-dependent stress-strain relation, a viscoelastic material does not have complete recov erability (that is, it does not have a unique equilibrium value). Anelasticity is a special case of viscoelasticity.

We simplify the stress-strain relation ( 3 ) by constructing symmetrized stresses and strains (Nowick and Berry, 1972). The symmetrized stresses (strairs), $\sigma_{i}\left(\epsilon_{i}\right)$, are linear combinations of $\tau_{i j}\left(e_{i j}\right)$ which remain unchanged under isotropic symmetry operations. The symmetrized stress-strain relations have the form $\sigma_{i}=J_{i} \epsilon_{i}$, where the $J_{i}^{\prime}$ 's are scalar quantities, which are constant for elastic media or a function of time for anelastic media. The symmetrized stresses for a three-dimensional isotropic solid are

$$
\begin{aligned}
& \sigma_{1}=\left(\tau_{11}+\tau_{22}+\tau_{33}\right), \\
& \sigma_{2}=\left(2 \tau_{11}-\tau_{22}-\tau_{33}\right), \\
& \sigma_{3}=\left(\tau_{22}-\tau_{33}\right), \\
& \sigma_{4}=\tau_{23}, \quad \sigma_{5}=\tau_{13}, \\
& \sigma_{6}=\tau_{12},
\end{aligned}
$$

and the symmetrized strains are

$$
\begin{aligned}
& \epsilon_{1}=\left(e_{11}+e_{22}+e_{33}\right), \\
& \epsilon_{2}=\left(2 e_{11}-e_{22}-e_{33}\right), \\
& \epsilon_{3}=\left(e_{22}-e_{33}\right), \\
& \epsilon_{4}=e_{23}, \quad \epsilon_{5}=e_{13}, \\
& \epsilon_{6}=e_{12} .
\end{aligned}
$$

The six resulting stress-strain relations for an isotropic elastic medium in terms of the symmetrized quantities are

$$
\begin{aligned}
& \sigma_{1}=3\left(\lambda+\frac{2}{3} \mu\right) \cdot \epsilon_{1}, \quad \sigma_{2}=2 \mu \cdot \epsilon_{2}, \\
& \sigma_{3}=2 \mu \cdot \epsilon_{3}, \quad \sigma_{4}=2 \mu \cdot \epsilon_{4}, \\
& \sigma_{5}=2 \mu \cdot \epsilon_{5}, \quad \sigma_{6}=2 \mu \cdot \epsilon_{6} .
\end{aligned}
$$

The first stress-strain relation corresponds to hydrostatic stresses and strains with the bulk modulus, $\kappa$, equal to $\lambda+2 \mu / 3$. The remaining five relations correspond to pure shear with the shear modulus equal to $\mu$.

Equations (7) represent naturally the behavior of both solids and fluids and lead to a convenient formulation for implicitly treating fluid-solid boundaries such as the seafloor. In a fluid the shear modulus $(\mu)$ is zero, the principle stresses $\left(\tau_{11}, \tau_{22}, \tau_{33}\right)$ are equal to each other and the shear stresses $\left(\tau_{12}, \tau_{23}, \tau_{13}\right)$ are zero. These conditions render the 
last five stress-strain relations in Eqs. (7) trivial. Hydrostatic pressure $(P)$, the volume change $(\Delta V / V)$, and the bulk modulus $(\kappa)$ are

$$
\begin{aligned}
& P=-\frac{1}{3}\left(\tau_{11}+\tau_{22}+\tau_{33}\right), \\
& \Delta V / V=e_{11}+e_{22}+e_{33}, \\
& \kappa=\lambda .
\end{aligned}
$$

The first stress-strain relation in (7) reduces to the fundamental relation in linear acoustics:

$$
P=-\kappa \cdot \Delta V / V,
$$

which states that the volumetric change of the fluid is proportional to the negative pressure. So general, lossy, isotropic fluids and anelastic, isotropic solids can be treated simultaneously by extending the concepts of bulk and shear moduli to two time-dependent functions.

Three approaches are used to measure the timedependent response of an anelastic material in quasistatic experiments (Nowick and Berry, 1972): (i) Creep is measured by applying a constant stress and observing the corresponding strain as a function of time. (ii) The elastic aftereffect or creep recovery is measured by releasing the stress applied in a creep experiment and measuring the strain as the material returns toward its original shape. (iii) The stress relaxation function is measured by applying a constant unit strain and observing the stress as a function of time. This is the approach we will use.

Associated with the stress relaxation function $M(t)$ are a number of quantities defined as follows:

$$
\begin{aligned}
& \text { Unrelaxed modulus, } M_{U}=M(0) \text {, } \\
& \text { relaxed modulus, } M_{R}=M(\infty) \text {, } \\
& \text { relaxation of the modulus, } \delta M=M_{U}-M_{R} \text {, } \\
& \text { relaxation strength, } \Delta=\delta M / M_{R},
\end{aligned}
$$

normalized stress relaxation function,

$$
\varphi(t)=\left[M(t)-M_{R}\right] / \delta M .
$$

The normalized stress relaxation function, $\varphi(t)$, is a monotonically decreasing function between unity (at $t=0$ ) and zero (at $t=\infty)$. The elastic stress-strain relations (7), which have the form $\sigma=J \epsilon$, can be generalized, using the stress relaxation function, to the time-dependent form:

$$
\begin{aligned}
\sigma(t) & =\int_{-\infty}^{t} M\left(t-t^{\prime}\right) d \epsilon\left(t^{\prime}\right) \\
& =\int_{-\infty}^{t} M\left(t-t^{\prime}\right) \frac{d \epsilon\left(t^{\prime}\right)}{d t^{\prime}} d t^{\prime},
\end{aligned}
$$

where $\sigma(t)$ and $\epsilon(t)$ are the stress and strain histories, respectively. Here, $M(t)$ is the stress history required to maintain a unit step function of strain. For isotropic media, represented by Eqs. (7), there would be a separate stress relaxation function corresponding to each of the bulk and shear moduli.
To relate the continuous, time domain stress-strain relations to the frequency domain we define the complex modulus, $M^{*}(\omega)$, by

$$
M^{*}(\omega)=\sigma(\omega) / \epsilon(\omega) .
$$

If the stress history in (11) is assumed to be sinusoidal with $\sigma=\sigma_{0} e^{i \omega t}$, by linearity the strain history will have the same frequency, and the strain will be delayed relative to the stress by a phase angle $\phi$ (that is $\epsilon=\epsilon_{0} e^{i(\omega t-\phi)}$ ). It can be shown that the real and imaginary parts of the complex modulus $\left[M_{1}(\omega)\right.$ and $M_{2}(\omega)$, respectively] are related to the stress relaxation function $M(t)$ by

$$
\begin{aligned}
& M_{1}(\omega)=M_{U}+\int_{0}^{\infty} \frac{d M(t)}{d t} \cos \omega t d t, \\
& M_{2}(\omega)=-\int_{0}^{\infty} \frac{d M(t)}{d t} \sin \omega t d t .
\end{aligned}
$$

Then the internal friction, or $Q^{-1}$, of the system is given (for $\phi<1$ ) by

$$
Q^{-1}=\phi \cong \tan \phi=M_{2} / M_{1},
$$

where $Q$ and $\phi$ are, in general, frequency dependent.

To determine the relationship between the complex modulus and the attenuation coefficient, consider a plane compressional wave, $u(x, t)$, propagating in the $x$ direction in a homogeneous media. This can be represented by the one-dimensional case of Eqs. (2), (4), and (11):

$$
\begin{aligned}
& \rho \ddot{u}=\sigma_{, x}, \\
& \sigma=\int_{-\infty}^{t} M\left(t-t^{\prime}\right) d \epsilon\left(t^{\prime}\right), \\
& \epsilon=u_{x},
\end{aligned}
$$

or, equivalently for harmonic motion,

$$
-\rho \omega^{2} u=M^{*} u_{, x x} .
$$

Here $M(t), \rho$, and $M^{*}$ are independent of $x$ (homogeneous media). Equation (16) is not strictly valid as a generalization of the anelastic wave equation, but it is appropriate for small attenuation (Nowick and Berry, 1972). A solution to this wave equation can be written as a damped wave:

$u(x, t ; \omega)=u_{0} \exp \{\alpha(\omega) x\} \exp \{i \omega[t-x / c(\omega)]\}$,

where $\alpha(\omega)$ is the attenuation coefficient and $c(\omega)$ is the phase velocity. Both $\alpha(\omega)$ and $c(\omega)$ are real functions in this case. Alternatively (17) can be written in terms of the complex wave number $K$ :

$$
\begin{aligned}
& u(x, t ; \omega)=u_{0} \exp \{i[\omega t-K(\omega) x]\}, \\
& K(\omega)=\omega / c(\omega)+i \alpha(\omega) .
\end{aligned}
$$

A complex wave number (or complex velocity) is frequently used to introduce attenuation into frequency domain approaches for solving the elastic wave equation (Fuchs and Müller, 1971, for example) and into solutions of the Helmholtz equation. Complex wave numbers (or complex velocities) are not appropriate for a time domain 
solution since the correct frequency dependence of these quantities cannot be introduced without specifically considering the transformed variables. Consequently, to introduce anelasticity into our time domain finite difference code, we use the time-dependent stress-strain relation [Eq. (11)] rather than the complex wave number [Eq. (18)].

By substituting the trial solution (18) into the generalized Helmholtz equation (16), we obtain the relationship between the complex wave number (attenuation and phase velocity) and the complex modulus (Aki and Richards, 1980):

$$
K(\omega)=\frac{\omega}{c(\omega)}+i \alpha(\omega)=\omega \sqrt{\frac{\rho}{M^{*}(\omega)}}
$$

In obtaining the complex wave number from the complex modulus, which is in turn obtained from the stress relaxation function, both causality and the necessary dispersion (frequency-dependent velocity) are guaranteed. The phase velocity and attenuation in Eq. (18) are not independent functions. To ensure causality in an anelastic medium, the phase velocity and attenuation must satisfy the KramersKrönig relations (Aki and Richards, 1980; Futterman, 1962). These relations can be obtained directly from the complex modulus, Eqs. (12) and (13), corresponding to any real stress relaxation function, Eqs. (10) and (11) (Nowick and Berry, 1972, p. 38). In obtaining the complex wave number from the complex modulus the relationship between phase velocity and attenuation is automatically constrained to satisfy the Kramers-Krönig relations.

It can also be shown from (18) that the $Q$, used to define the internal friction (14), is the spatial $Q$ given by (Aki and Richards, 1980, p. 178):

$$
\frac{1}{Q(\omega)}=\frac{2 c(\omega) \alpha(\omega)}{\omega} .
$$

\section{TWO MODELS FOR ANELASTIC MEDIA}

The stress relaxation function can represent a broad range of anelastic behavior and for most applications further simplifying assumptions are made. We discuss here two models. The standard anelastic solid is an appropriate model for many individual relaxation mechanisms but it yields a strongly frequency-dependent $Q$. [Unless otherwise indicated all of the material discussed here on the standard anelastic solid is from Nowick and Berry (1972, pp. 4657)]. However it is commonly observed in seismology and marine geoacoustics that $Q$ is independent of frequency over a broad bandwidth (Hamilton, 1976a,b). To explain the broadband behavior in seismology, Liu et al (1976) proposed a constant $Q$ absorption band model based on a continuous, box distribution of relaxation mechanisms. In this section we present briefly these two models and compare their $Q$ spectra. We show that the standard anelastic solid gives a highly variable $Q$ over the bandwidth of our finite difference calculations. The constant $Q$ absorption band model gives a much flatter $Q$ spectrum and it is the model that we use in the finite difference algorithm.
The mechanical model for the standard anelastic solid is represented by the linear differential stress-strain equation:

$$
\sigma+\tau_{\epsilon} \dot{\sigma}=M_{R}\left(\epsilon+\tau_{\sigma} \dot{\epsilon}\right)
$$

where $\tau_{\sigma}$ is the relaxation time at constant stress and $\tau_{\epsilon}$ is the relaxation time at constant strain. The two relaxation times are related by

$$
\tau_{\epsilon}=\left(M_{R} / M_{U}\right) \tau_{a},
$$

and the stress relaxation function is

$$
\begin{aligned}
M(t) & =M_{R}+\left(M_{U}-M_{R}\right) \exp \left(-t / \tau_{\epsilon}\right) \\
& =M_{R}\left[1+\left(\tau_{\sigma} / \tau_{\epsilon}-1\right) \exp \left(-t / \tau_{\epsilon}\right)\right] .
\end{aligned}
$$

In the frequency domain the real and irnaginary parts of the complex modulus are

$$
\begin{aligned}
& M_{1}(\omega)=M_{U}-\frac{\delta M}{1+\omega^{2} \tau_{\epsilon}^{2}}, \\
& M_{2}(\omega)=\delta M \frac{\omega \tau_{\epsilon}}{1+\omega^{2} \tau_{\epsilon}^{2}} .
\end{aligned}
$$

For the standard anelastic solid the internal friction [from Eqs. (10), (14), and (24)] is

$$
Q(\omega)^{-1} \cong \frac{\Delta}{(1+\Delta)^{1 / 2}} \frac{\omega \bar{\tau}}{1+\omega^{2} \bar{\tau}^{2}}=\frac{\omega\left(\tau_{\sigma}-\tau_{\epsilon}\right)}{1+\omega^{2} \tau_{\sigma} \tau_{\epsilon}},
$$

where $\bar{\tau} \equiv\left(\tau_{\sigma} \tau_{\epsilon}\right)^{1 / 2}$. For $\Delta<1$, the minimum $Q\left(Q_{0}\right)$ is $2 / \Delta$ and it occurs at $\omega=1 / \tau$. The phase velocity, $c(\omega)$, for the standard anelastic solid is given by (Aki and Richards, 1980):

$$
[c(\omega)]^{2}=\frac{M_{U}}{\rho}\left[1+\left(\frac{M_{U}}{M_{R}}-1\right) \frac{1}{1+\omega^{2} \vartheta_{\sigma}^{2}}\right]^{-1} .
$$

Note that Aki and Richards (1980) and Liu et al. (1976) refer to the standard anelastic sclid as the "standard linear solid." They also switch the subscripts of the relaxation times. In their notation, the relaxation time of strain at constant stress is $\tau_{\epsilon}$ and the relaxation time of stress at constant strain is $\tau_{\sigma}$. In this section we use the nomenclature of Nowick and Berry (1972).

Figure 1 shows the $Q$ spectra for the standard anelastic solid. Over the bandwidth of the source used in our finite difference formulation (approximately 2 to $25 \mathrm{~Hz}$ ) $Q$ varies from about 20 to 50 , which is unacceptable.

To broaden the frequency-independent bandwidth of $Q$, we consider the constant $Q$ absorption band model, based on a continuous distribution of relaxation mechanisms (Liu et al., 1976). The distribution of relaxation mechanisms is represented by a normalized relaxation spectrum, $\Phi(\ln \tau)$, defined by (Nowick and Berry, 1972, pp. 77-80): 

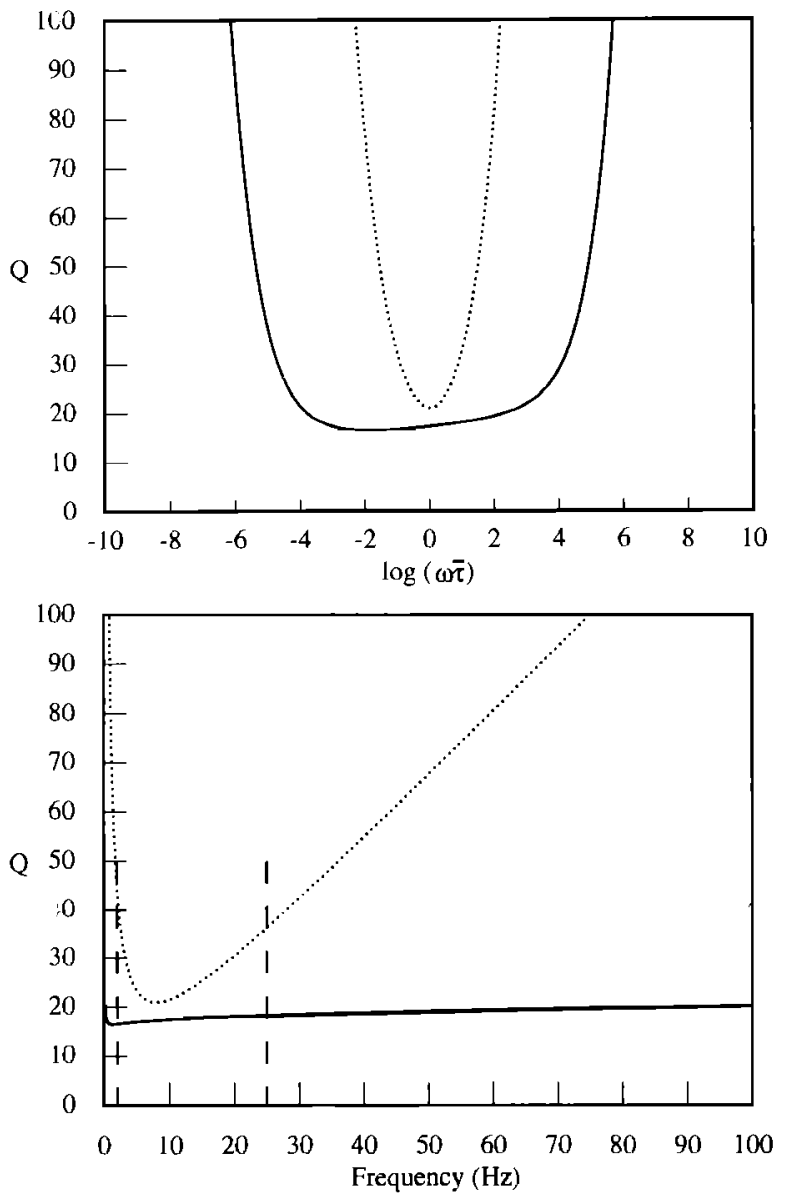

FIG. 1. The $Q$ spectra are plotted as a function of normalized angular frequency (top) and as a function of unnormalized frequency (bottom) for the standard anelastic solid (dashed) and for the constant $Q$ absorption band model (solid). The $40-\mathrm{dB}$ down points of the source in our finite difference formulation are about 2 and $25 \mathrm{~Hz}$. In this band, $Q$ is highly dependent on frequency for the standard elastic solid. The constant $Q$ absorption band model increases the bandwidth over which $Q$ is relatively frequency independent and is a much more acceptable model. The spectrum for the standard anelastic solid is based on Eq. (25) with $\tau_{\sigma}$ and $\tau_{\epsilon}$ equal to 2 and $0.0002 \mathrm{~s}$, respectively, and a $Q_{0}$ of 20 . The spectrum for the constant $Q$ absorption band model is based on Eqs. (14), (30), and (31) with $\tau_{1}$ and $\tau_{2}$ equal to 0.0002 and $2 \mathrm{~s}$, respectively, and a $Q_{0}$ of 20 .

$$
\begin{gathered}
\phi(t)=\int_{-\infty}^{\infty} \Phi(\ln \tau) e^{-t / \tau} d(\ln \tau), \\
\int_{-\infty}^{\infty} \Phi(\ln \tau) d(\ln \tau)=1 .
\end{gathered}
$$

In these expressions $\tau$ represents the relaxation time at constant strain, $\tau_{\epsilon}$. The subscript has been dropped for convenience. It is convenient and traditional to use a logarithmic scale for time and frequency. For example, physically realistic relaxation spectra span many orders of magnitude. Also for the standard anelastic solid the internal friction is a symmetric function when plotted against the logarithm of frequency. Before applying or plotting functions, the argument of the logarithm is normally converted to a ratio, $\tau / \tau_{m}$ or $\omega \tau_{m}$, where $\tau_{m}$ is some reference value.

If the normalized relaxation spectrum is a box distribution with a broad enough bandwidth, then $Q$ will be approximately independent of frequency near the middle of
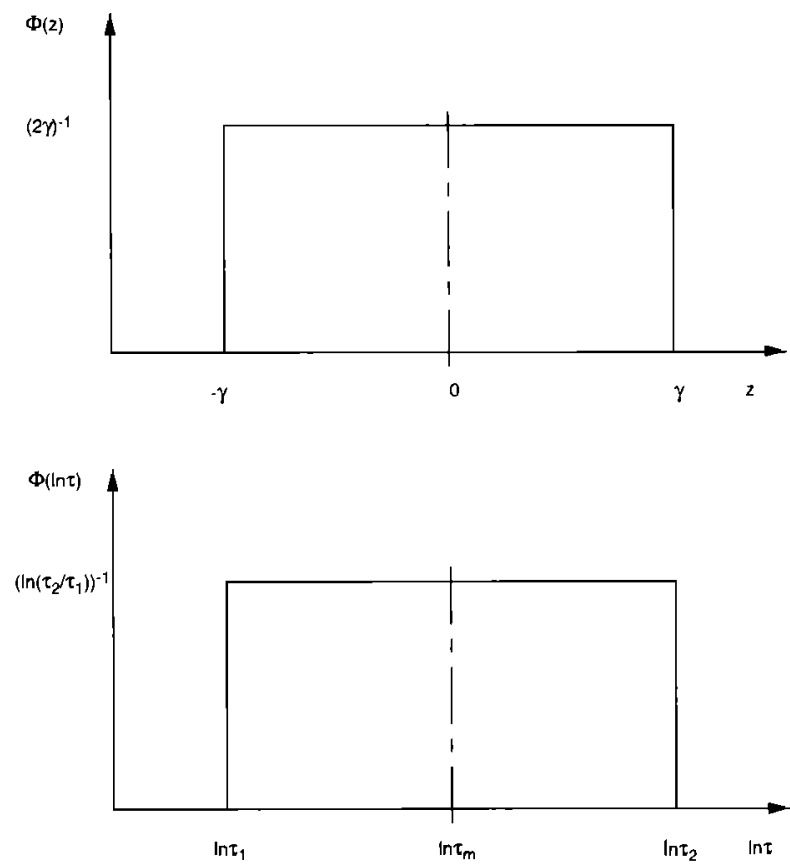

FIG. 2. The normalized relaxation spectrum, $\Phi(\ln \tau)$, used by Day and Minster (1984), is the box distribution shown in the lower frame. Nowick and Berry (1972) use the same distribution, $\Phi(z)$ with $z=\ln \left(\tau / \tau_{m}\right)$, as shown in the top frame. This relaxation spectrum is the basis of the constant $Q$ absorption band model used in the 2-D finite difference code presented in the paper.

the band (Day and Minster, 1984; Liu et al., 1976). The normalized box relaxation spectrum is (Nowick and Berry, 1972):

$$
\Phi(z)=\left\{\begin{array}{l}
0, \text { for } \quad z>\gamma \\
(2 \gamma)^{-1}, \quad \text { for }-\gamma \leqslant z \leqslant \gamma, \\
0, \text { for } \quad z<-\gamma,
\end{array}\right.
$$

where the time and frequency variables are scaled to

$$
\begin{aligned}
& z \equiv \ln \left(\tau / \tau_{m}\right), \\
& x \equiv \ln \left(\omega \tau_{m}\right) .
\end{aligned}
$$

The spectrum as a function of $\ln \tau$ and as a function of the normalized variable, $z$, is shown in Fig. 2. The normalization value, $\ln \tau_{m}$, is the midpoint of $\Phi(\ln \tau)$ and $\gamma$ is the half-width of $\Phi(z)$. The corresponding components of the operational modulus are given by the following relations:

$$
\begin{aligned}
& M_{1}=M_{R}+\frac{\delta M}{4 \gamma} \ln \left(\frac{1+e^{2(x+\gamma)}}{1+e^{2(x-\gamma)}}\right), \\
& M_{2}=\frac{\delta M}{2 \gamma}\left[\tan ^{-1} e^{(x+\gamma)}-\tan ^{-1} e^{(x-\gamma)}\right] .
\end{aligned}
$$

For $\tau_{2}^{-1}<\omega<\tau_{1}^{-1}$ (see Fig. 2), the internal friction of the constant $Q$ absorption band model is approximately (Day and Minster, 1984):

$$
Q^{-1} \approx \frac{\pi}{2} \frac{\delta M}{M_{U}}\left(\ln \frac{\tau_{2}}{\tau_{1}}\right)^{-1}=\frac{\pi}{2} \frac{\delta M}{M_{U}}(2 \gamma)^{-1}=Q_{0}^{-1} .
$$

The $Q$ spectrum for the constant $Q$ absorption band model [from Eqs. (14) and (30)] is compared to the $Q$ spectrum 
for the standard anelastic solid [Eq. (25)] in Fig. 1. The constant $Q$ absorption band model gives a much flatter $Q$ over the bandwidth of the finite difference source (2-25 $\mathrm{Hz}$ ).

\section{THE FINITE DIFFERENCE FORMULATION}

We have implemented the anelasticity formulation for the constant $Q$ absorption band model into our finite difference code for a two-dimensional Cartesian geometry. For the two-dimensional case the "symmetrized" stresses and strains [Eqs. (5) and (6)] become

$$
\begin{array}{lll}
\sigma_{1}^{\prime}=\left(\tau_{11}+\tau_{22}\right), & \sigma_{2}^{\prime}=\left(\tau_{11}-\tau_{22}\right), & \sigma_{6}^{\prime}=\tau_{12}, \\
\epsilon_{1}^{\prime}=\left(e_{11}+e_{22}\right), & \epsilon_{2}^{\prime}=\left(e_{11}-e_{22}\right), & \epsilon_{6}^{\prime}=e_{12},
\end{array}
$$

where a prime is used on the symmetrized quantities to distinguish the two-dimensional problem from the threedimensional problem. The corresponding stress-strain relations for a two-dimensional, isotropic, elastic medium in terms of the symmetrized quantities are

$$
\begin{aligned}
& \sigma_{1}^{\prime}=2(\lambda+\mu) \cdot \epsilon_{1}^{\prime}, \\
& \sigma_{2}^{\prime}=2 \mu \cdot \epsilon_{2}^{\prime}, \\
& \sigma_{6}^{\prime}=2 \mu \cdot \epsilon_{6}^{\prime} .
\end{aligned}
$$

Anelasticity is introduced by generalizing each of these three stress-strain relations to a convolution of the rate of strain with a time-dependent stress relaxation function [Eq. (11)]. For the constant $Q$ absorption band model, the time-dependent stress-strain relations can be approximated by (Day and Minster, 1984):

$$
\sigma(t)=M_{U}\left(\epsilon(t)-\sum_{i=1}^{n} \zeta_{i}(t)\right),
$$

where the $\xi_{i}$ satisfy

$$
\frac{d \zeta_{i}(t)}{d t}+v_{i} \zeta_{i}(t)=\left(\frac{\tau_{1}^{-1}-\tau_{2}^{-1}}{\pi} w_{i} Q_{0}^{-1}\right) \epsilon(t)
$$

and

$$
\begin{aligned}
& v_{i}=\frac{1}{2}\left[l_{i}\left(\tau_{1}^{-1}-\tau_{2}^{-1}\right)+\left(\tau_{1}^{-1}+\tau_{2}^{-1}\right)\right], \\
& w_{i}=\frac{2}{n P_{n-1}\left(l_{i}\right) P_{n}^{\prime}\left(l_{i}\right)},
\end{aligned}
$$

where $\tau_{1}$ and $\tau_{2}$ are the bounding relaxation times used in Eqs. (28) and (31) and in Fig. 2. The $l_{i}$ and $w_{i}$ are the abscissas and weight factors for Gaussian integration (Abramowitz and Stegun, 1965, p. 916). Following Day and Minster, we used a five pole approximant $(n=5)$.

The unrelaxed moduli, $M_{U}$, in Eq. (34) are the same moduli that appear in the stress-strain relations (33). Lamé's parameters $\lambda$ and $\mu$ in (33) are determined from the nominal velocities for elastic media, $V_{P}$ and $V_{S}$, and density, $\rho$, by

$$
\begin{aligned}
& \lambda=\rho\left(V_{P}^{2}-2 V_{S}^{2}\right), \\
& \mu=\rho V_{S}^{2} .
\end{aligned}
$$

The wave velocities for infinite $Q$ media, computed by this formulation, will correspond to the nominal velocities for elastic media. When $Q$ is finite, the wave velocities will differ from the nominal values for elastic media (and will be frequency dependent) because of the dispersion associated with anelasticity [Eq. (19)].

Our finite difference formulation solves the twodimensional wave equation for heterogeneous, isotropic, anelastic media in terms of displacements using the offset grid scheme proposed by Virieux (1986). At each grid point the medium is represented by nominal compressional and shear velocities, density, and compressional and shear attenuation. The width of the absorption band, determined by $\tau_{1}$ and $\tau_{2}$, is constant for a given model (Fig. 2). Also at each grid point we solve for the vertical and horizontal particle displacement by time stepping through the following algorithm. Symmetrized strains are computed from (4) and (32). For each of the three stress-strain relations, (33), there are five internal variables, $\zeta_{i}$ 's, which are obtained from the symmetrized strains by a finite difference approximation to Eq. (35). So at each grid point there are twenty-two variables: Five medium parameters, two field variables (displacements) and fifteen internal variables. The symmetrized stresses $\left(\sigma_{i}\right)$ are determined from the symmetrized strains $\left(\epsilon_{i}\right)$ and $\zeta_{i}$ 's by (34). The stresses $\left(\tau_{i}\right)$ are determined from the symmetrized stresses $\left(\sigma_{i}\right)$ by Eq. (32) and the updated displacements $\left(u_{i}\right)$ are computed from $\mathrm{Eq}$. (2).

\section{VALIDITY CHECK FOR A HOMOGENEOUS FLUID}

To confirm that the results of the finite difference code are valid, we first consider propagation in a twodimensional, homogeneous, fluid with and without losses. The layout for our tests is shown in Fig. 3. The response of a point source is observed on a line of pressure receivers directly below the source. The source tirne series in pressure is the third derivative of a Gaussian curve with a peak frequency of $10 \mathrm{~Hz}$ (Stephen et al., 1985). Although the finite difference solution scales to periods (for time) and wavelengths (for space), we use specific frequencies and ranges in this example for convenience.

$Q$ is obtained from impulsive source data at two receivers by the "spectral ratio method" (Ganley and Kanasewich, 1980, for example). From Eq. (17) the natural logarithm of the ratio of two amplitude spectra from ranges $x_{1}$ and $x_{2}$ is

$$
\ln \left(\frac{\left|u\left(x_{2}, \omega\right)\right|}{\left|u\left(x_{1}, \omega\right)\right|}\right)=-\alpha\left(x_{2}-x_{1}\right)
$$

So the attenuation parameter, $\alpha$, and $Q$, can be obtained from the slope, $m$, of the logarithm of the spectral ratios plotted as a function of frequency:

$$
\begin{aligned}
& \alpha=m f /\left(x_{2}-x_{1}\right), \\
& Q=\pi\left(x_{2}-x_{1}\right) /(\mathrm{cm}) .
\end{aligned}
$$

Note that since $c$ is slightly dependent on frequency, constant $Q$ does not exactly correspond to $a$ proportional to frequency. This method has the advantage that it is not 


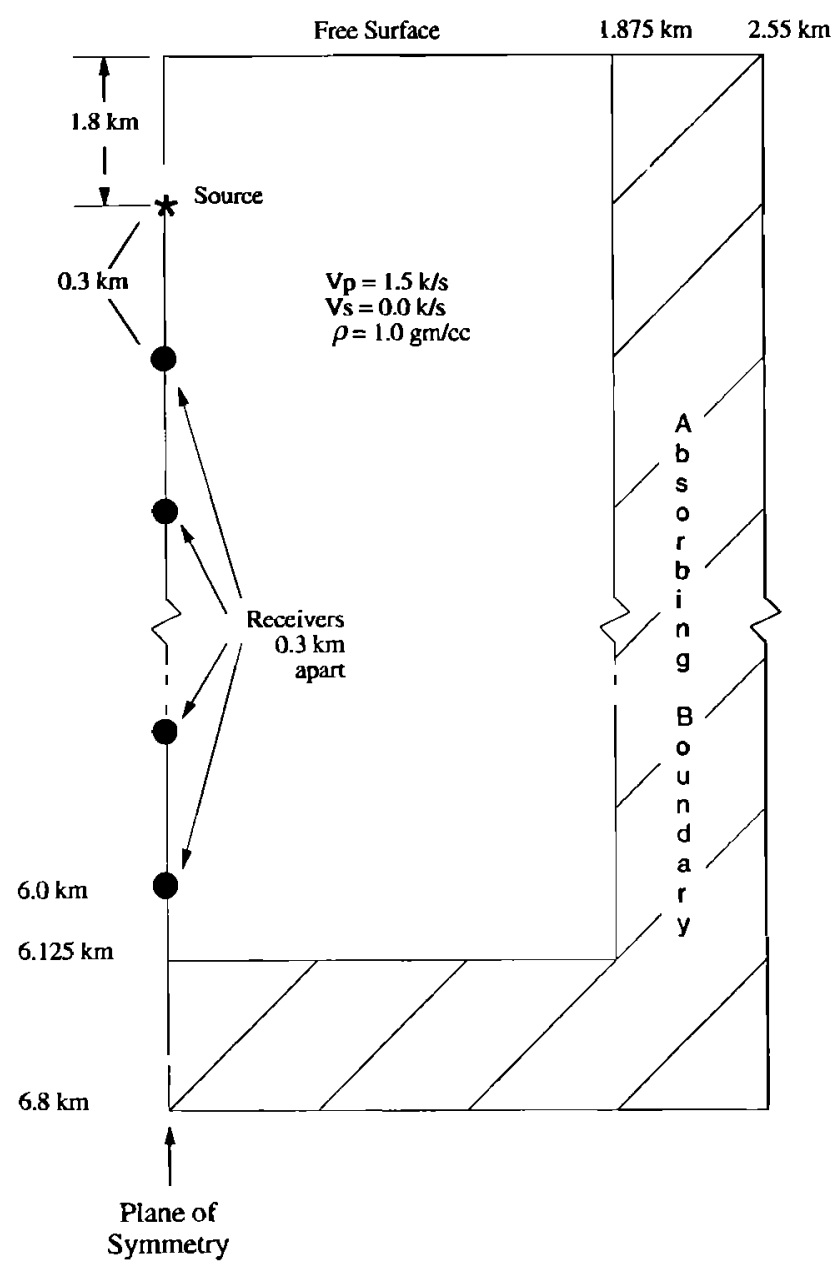

FIG. 3. Layout of the finite difference grid for the models used to test the two-dimensional anelastic finite difference code for homogeneous fluids. The source is proportional to the third derivative of a Gaussian in pressure with a peak frequency at $10 \mathrm{~Hz}$. At $10 \mathrm{~Hz}$ the grid has 20 points per wavelength. Grid dispersion is reduced with more points per wavelength (Alford et al, 1974; Virieux, 1986).

necessary to consider the effects of spreading loss and reflection and transmission at interfaces provided these effects are frequency independent.

As a reference, we first consider a lossless fluid $\left(Q_{P}=\infty\right)$. The computed spreading loss coefficient for the two-dimensional finite difference code based on rms levels is $10.01 \mathrm{~dB}$, which is in excellent agreement with the theoretical geometrical loss coefficient for a line source of 10 dB. Figure 4(a) shows waveforms at 2.1- and 3.9-km depth for a medium with no attenuation. The corresponding spectra [Fig. 4(b)] show that the peak frequency is 10 $\mathrm{Hz}$ with $40-\mathrm{dB}$ down points at about 2 and $25 \mathrm{~Hz}$. Spectral shape remains constant with depth within the 40-dB down points and the computed $Q$ based on the spectral ratio technique [Fig. 4(c)] is 5237, in good agreement with an infinite $Q$.

The waveforms for a lossy fluid with a $Q_{P}$ of 20 ( $\tau_{1}$ and $\tau_{2}$ equal to 0.0002 and $2.0 \mathrm{~s}$, respectively) are shown in Fig. $5(a)$. Figure $5(b)$ shows the corresponding spectra after correction for geometrical spreading and there is greater loss at high frequencies for the farther receiver. Because of
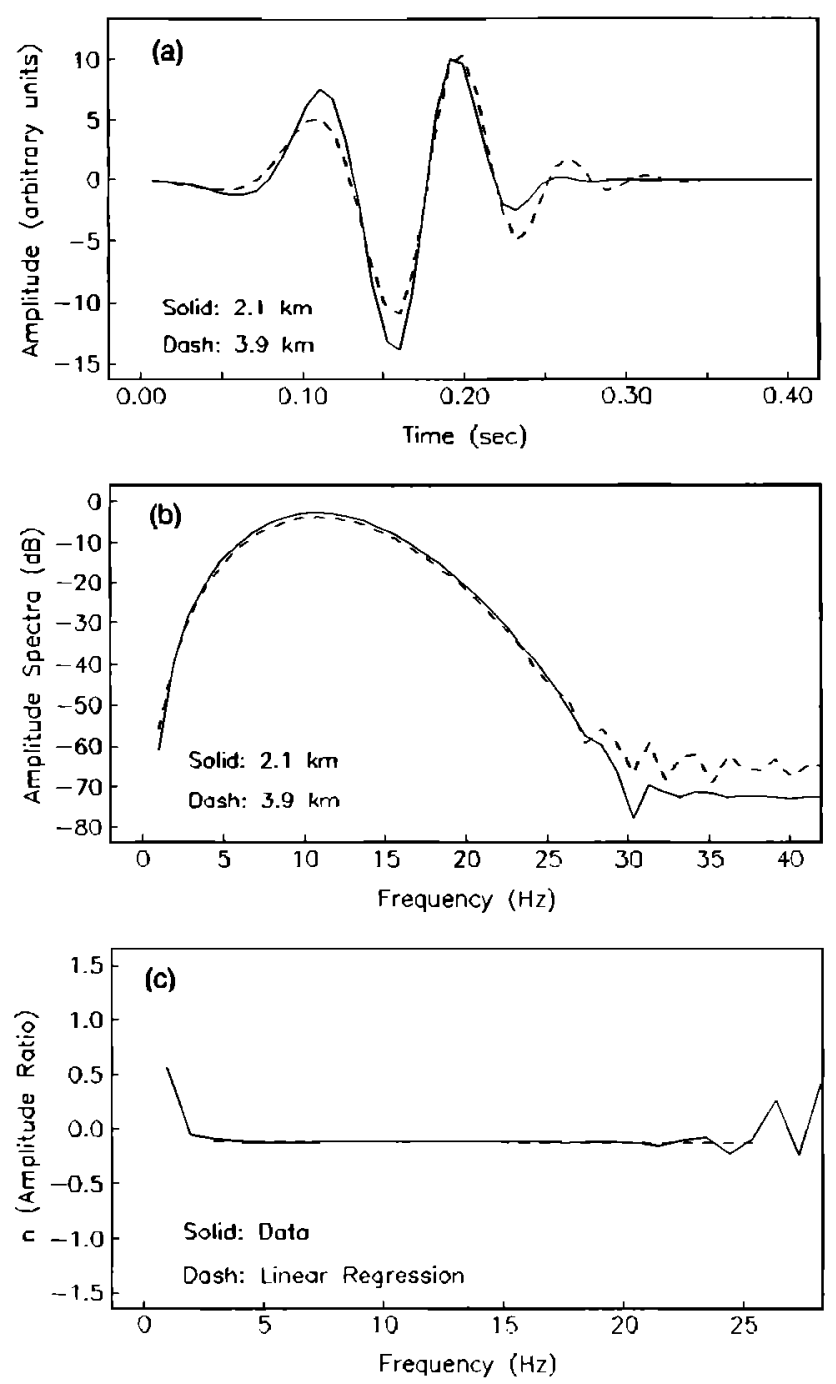

FIG. 4. The waveforms at 2.1 and $3.9 \mathrm{~km}$ are compared in (a) for a two-dimensional fluid model with no attenuation. The $3.9-\mathrm{km}$ waveform (dashed) shows coda resulting from grid dispersion at higher frequencies. The amplitudes decay due to geometrical spreading at $10 \log R$. (b) shows the spectra of the waveforms in (a) after correction for geometrical spreading. The spectra are identical down to $40 \mathrm{~dB}$ indicating perfectly elastic behavior. The 40-dB down points are approximately 2 and $25 \mathrm{~Hz}$. (c) shows the spectral ratios for the wave forms in (a). The slope is essentially zero giving a $Q$ of -5237 which is in good agreement with infinite $Q$ for short range propagation.

the attenuation, the upper $40-\mathrm{dB}$ down point has been reduced to $16 \mathrm{~Hz}$. The spectra are well within the constant $Q$ absorption band (Fig. 1). Figure 5(c) shows the spectral ratios. The attenuation is approximately linear between the 40-dB down points and the computed $Q$ is 19.60 based on a least-squares fit. This is within $2 \%$ of the input value and confirms the validity of the code for homogeneous fluids.

\section{VALIDITY CHECK FOR A LOSSLESS FLUID OVER AN ANELASTIC SOLID}

To check the code for a seafloor model, we consider a lossless fluid over an elastic or anelastic solid as outlined in Fig. 6. The source is the same as in the previous example.

For receivers in the bottom we record time series of the effective pressure or normalized dilatation, $-(\lambda$ $+2 \mu) \nabla \cdot u$. This quantity is proportional to the square root 

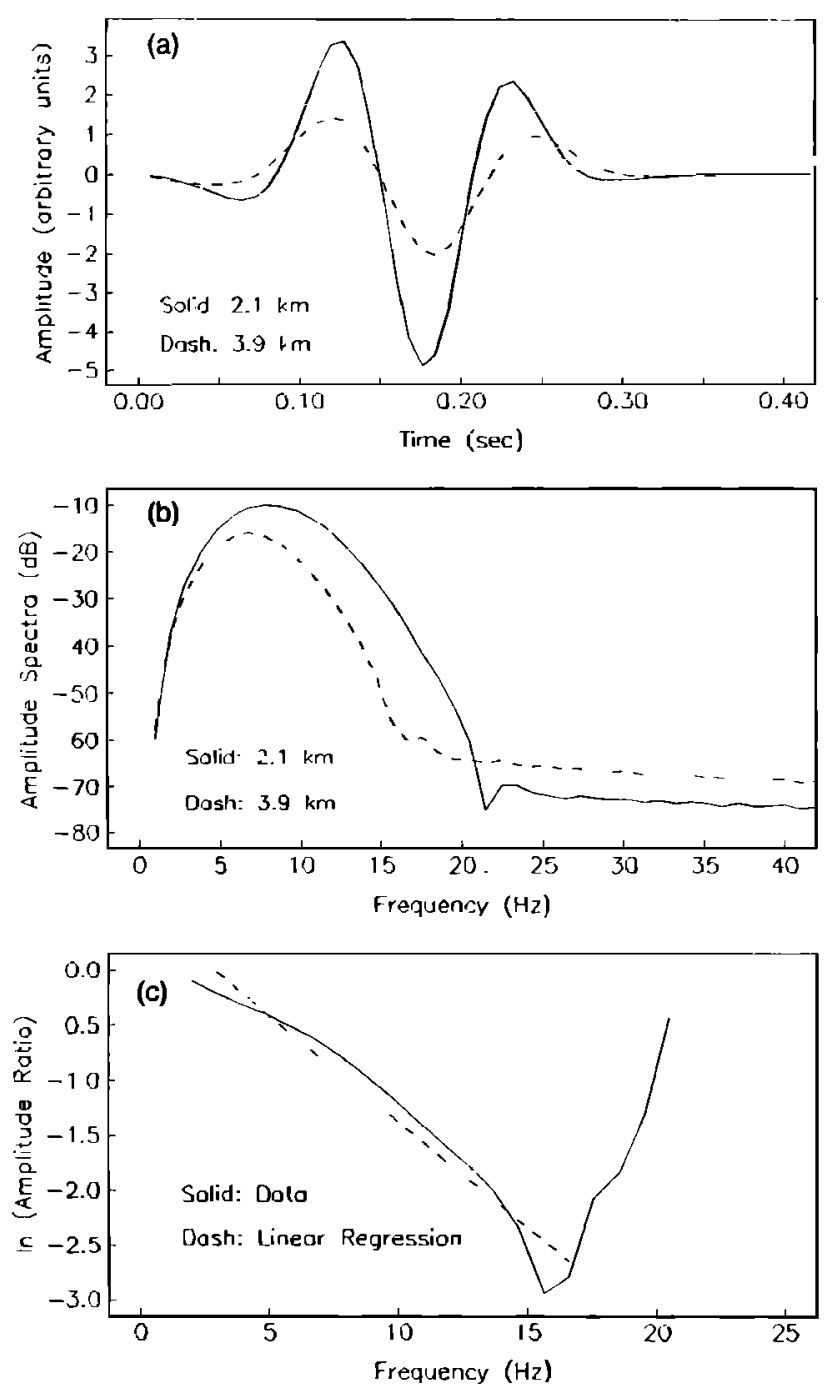

FIG. 5. Waveforms at 2.1 and $3.9 \mathrm{~km}$ for a two-dimensional fluid model with a $Q_{0}$ of 20 ( $\tau_{1}$ and $\tau_{2}$ equal to 0.0002 and $2.0 \mathrm{~s}$, respectively) are shown in (a). Grid dispersion is less apparent in the anelastic case [compare with Fig. 4(a)] since the high frequencies have been attenuated. The amplitudes are much less at $3.9 \mathrm{~km}$ because of the attenuation. (b) shows the spectra of the wave forms in (a) after correction for geometrical spreading. The decrease in amplitude with increasing frequency is quite apparent. The peak signal level is about $50 \mathrm{~dB}$ above the numerical noise. The 40-dB down points for the farther receiver are 2 and $15 \mathrm{~Hz}$. (c) shows the spectral ratios for the wave forms in (a). The best fit to the line between the $40-\mathrm{dB}$ down points gives a $Q$ of 19.6. This is within $2 \%$ of the nominal $Q$ value and is within the range of $Q$ values for the constant $Q$ absorption band model (Fig. 1).

of the compressional energy density, with the sign of the particle motion preserved. It has the advantage of separating compressional effects from shear effects and it reduces directly to pressure when the shear modulus vanishes.

Figure 7 shows the time series, spectra, and spectral ratios, respectively, for the case of an elastic bottom $\left(Q_{P}=Q_{S}=\infty\right)$. The computed $Q_{P}$ is -3030 in good agreement with the input value.

Similarly, Fig. 8 shows the time series, spectra and spectral ratios for the case of an anelastic bottom $\left(Q_{P}=Q_{S}=20\right)$. The time series show the spreading and decreased frequency content of the trace at greater depth. The amplitude spectra show that progressively higher fre-

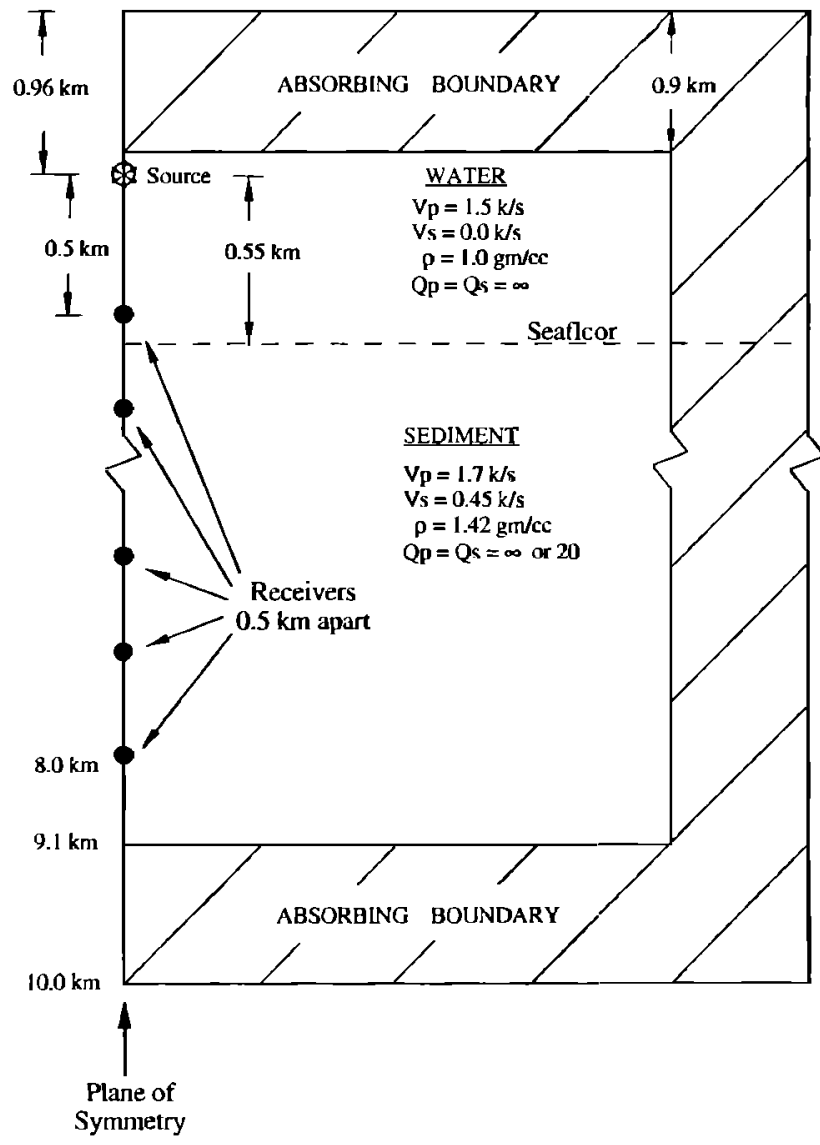

FIG. 6. Layout of the finite difference grid for the rr odels used to test the code for a lossless fluid over an anelastic solid. The source is $0.55 \mathrm{~km}$ above the seafloor and the effective pressure, $-(\lambda+2 \mu) \nabla \cdot u$, is observed in the solid directly below the source.

quencies are losing more energy at greater depth. The spectral ratio method, applied over the band with good signalto-noise gives a $Q_{P}$ of 20.62 which is again in good agreement with the input value. For this geometry, at normal incidence, there is no compressional to shear conversion at the seafloor and there are no shear wave arrivals on which to compute $Q_{S}$.

Figure 9 shows a snapshot of the normalized compressional and shear fields (Dougherty and Stephen, 1988) for a line source over a flat, homogeneous, sedimentary bottom with attenuation. The point source (in two-dimensional Cartesian coordinates) is excited in water $\left(V_{P}=1.5 \mathrm{k} / \mathrm{s}\right.$, $\left.\rho=10^{3} \mathrm{~kg} / \mathrm{m}^{3}, Q_{P}=\infty\right), 550 \mathrm{~m}$ above a flat, homogeneous, sedimentary seafloor $\left(V_{P}=1.7 \mathrm{k} / \mathrm{s}, \quad V_{S}=0.45 \mathrm{k} / \mathrm{s}\right.$, $\left.\rho=1.42 \times 10^{3} \mathrm{~kg} / \mathrm{m}^{3}, Q_{P}=20, Q_{S}=20\right)$. Direct, reflected, transmitted $(P$ and $S$ ) and head $(P$ and $S$ ) waves can be identified. The wave types for a similar model without attenuation are discussed in more detail in Stephen (1991).

In Fig. 10 we compare the differences in the compressional wave field for models with and without attenuation. The compressional body wave in the bottom and the $P$ head wave in the water are obviously atten uated. In Fig. 11 we compare the time series, with and without attenuation, for a line of pressure receivers on the seafloor. The direct/ reflected water waves are identical in the 1wo cases except at the critical point. The compressional head wave however 

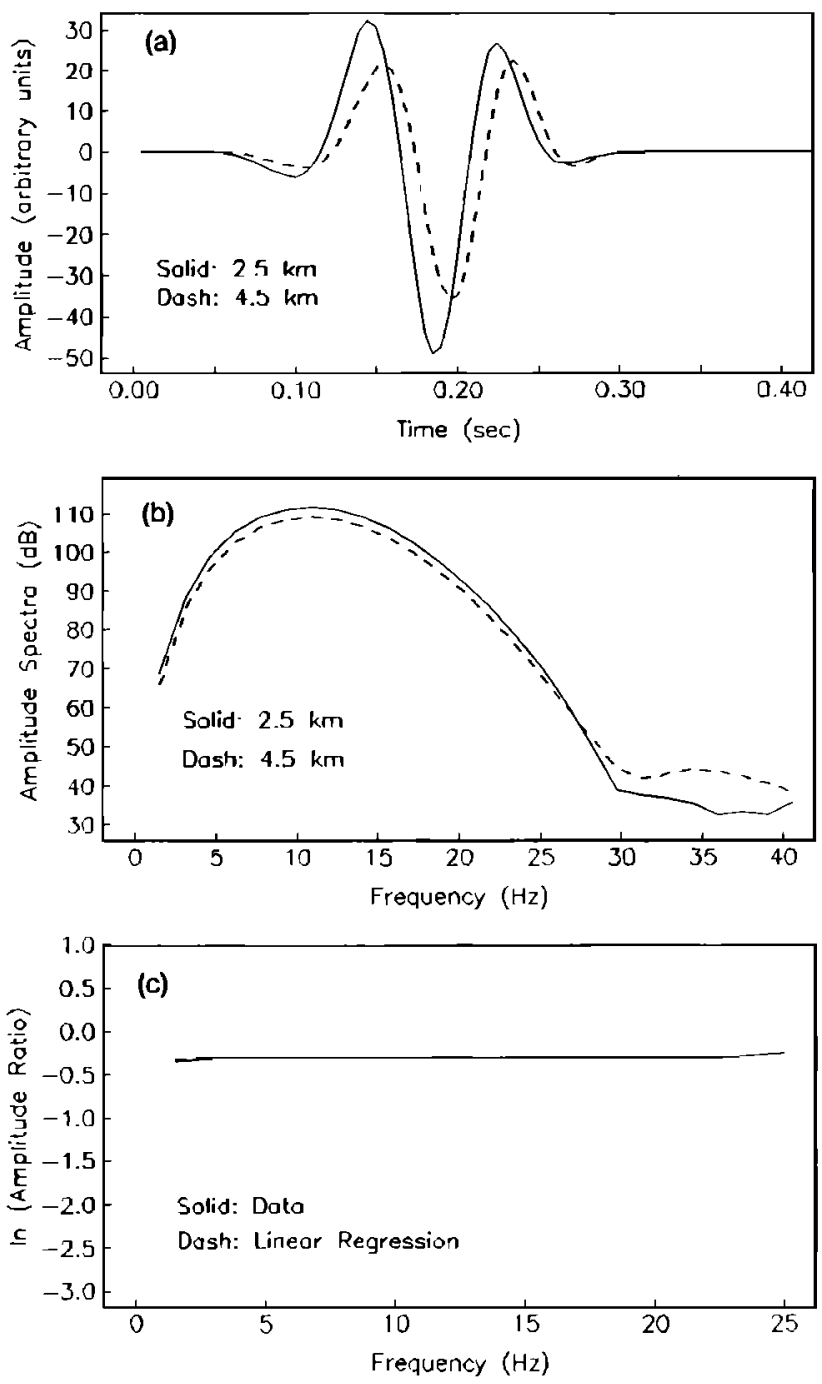

FIG. 7. The waveforms at 2.5 and 4.5-km depth are compared in (a) for the two-dimensional fluid/solid model, described in Fig. 6, with no attenuation. (b) The spectra of the waveforms in (a). (c) The spectral ratios for the waveforms in (a). The slope is essentially zero giving a $Q$ of -3030 which is in good agreement with infinite $Q$ for short range propagation.

shows strong attenuation with range for the anelastic case.

We did not compute a $Q$ from the compressional head wave because, near the critical point, the head wave is frequency dependent even for elastic media. This violates the assumption, in the spectral ratio method, that all of the frequency dependence is caused by intrinsic attenuation. In applying the spectral ratio method to real data, care must be taken to ensure that frequency dependent effects other than intrinsic attenuation are not contaminating the results. For example the steep velocity gradients frequently observed with depth below the seafloor will introduce frequency dependent propagation effects that are not associated with attenuation.

\section{CONCLUSIONS}

We have developed a capability to study intrinsic attenuation and scattering at seafloors with both surface roughness and volume heterogeneities. The method solves
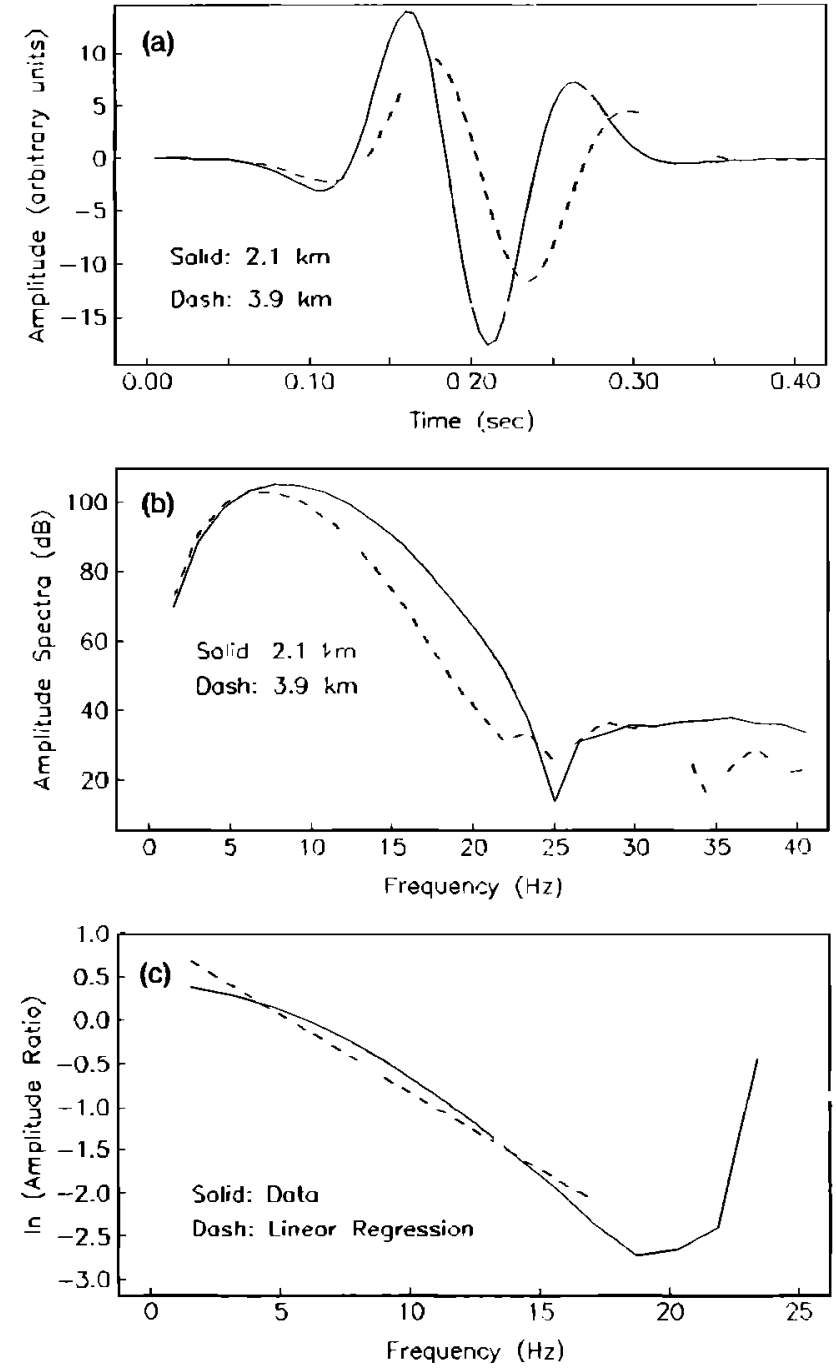

FIG. 8. (a) The waveforms at 2.5 and $4.5 \mathrm{~km}$ for the two-dimensional fluid/solid model in Fig. 6 with a $Q$ of 20 for both compressional and shear waves ( $r_{1}$ and $\tau_{2}$ equal to 0.0002 and $2.0 \mathrm{~s}$, respectively). (b) The spectra of the waveforms in (a). The decrease in amplitude with increasing frequency is quite apparent. The spectral ratios for this model [(c)] give a $Q$ of 20.62 which is in good agreement with the input value.

the anelastic wave equation in the time domain in twodimensional Cartesian coordinates by the method of finite differences.

The formulation treats lossless/lossy fluids and elastic/anelastic solids simultaneously. Lossy fluids are correctly represented by the anelastic formulation when the shear modulus is set to zero. Fluid-solid boundaries can be treated implicitly. The formulation for lossy media is based on stress relaxation functions for the compressional and shear components of the symmetrized stressstrain relations. The method is carried out entirely in the time domain, but the approach is consistent with the complex velocity (or complex wave number) technique which is commonly used for frequency domain solutions.

The finite difference algorithm is based on the constant $Q$ absorption band model (Liu et al., 1976; Nowick and Berry, 1972) and on the Padé approximant method outlined by Day and Minster (1984). The approach satisfies 


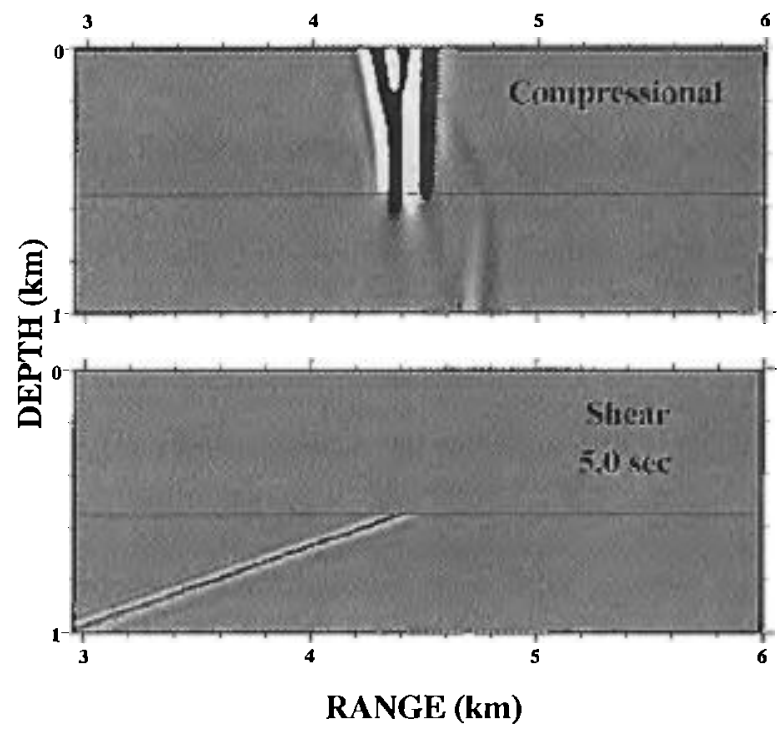

FIG. 9. The compressional (div) and shear (crl) snapshots at $5.0 \mathrm{~s}$ after a point source is excited over a flat, homogeneous sedimentary bottom with a $Q$ of 20 for both compressional and shear waves. Direct, reflected, transmitted ( $P$ and $S$ ), and head $(P)$ waves can be identified. [The wave types for a similar model without attenuation are discussed in more detail in Stephen (1991).]

the Kramers-Krönig relations and causality and the correct dispersion for a given attenuation model are ensured.

This method can be used in a forward modeling mode to identify characteristics in geoacoustic data which will distinguish between intrinsic attenuation and scattering. Some questions that can be addressed include: Given that the seafloor is rough and heterogeneous on a broad range of scales is scattering alone sufficient to explain observed attenuations? What effects will intrinsic attenuation have on scattering mechanisms? What does "frequency-

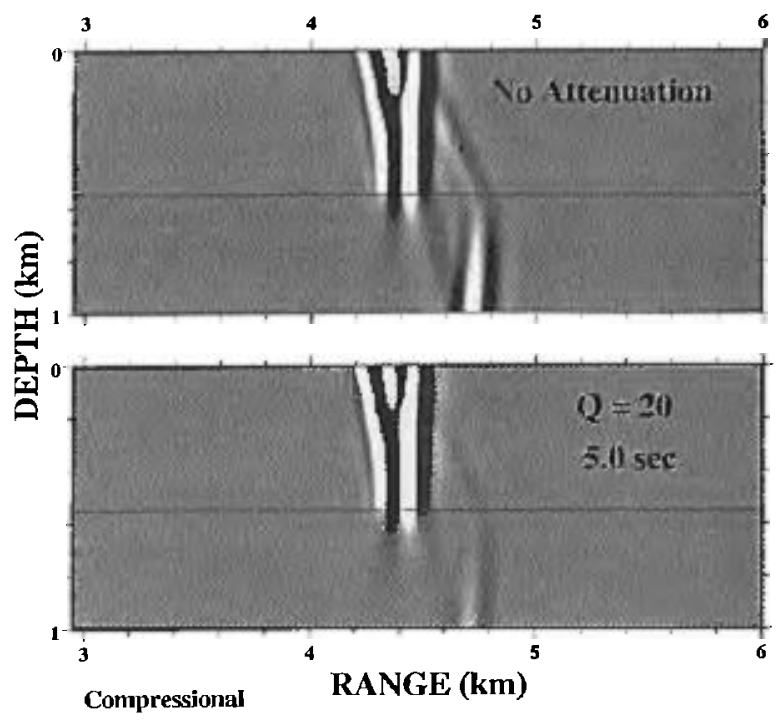

FIG. 10. The compressional snapshots at $5.0 \mathrm{~s}$ are compared for models with and without attenuation in the bottom. The attenuation snapshot is the same as the compressional snapshot in Fig. 9. The compressional body wave in the bottom and the compressional head wave in the water are clearly affected by the attenuation. The direct compressional body wave in the water is, of course, unaffected by the attenuation in the bottom.
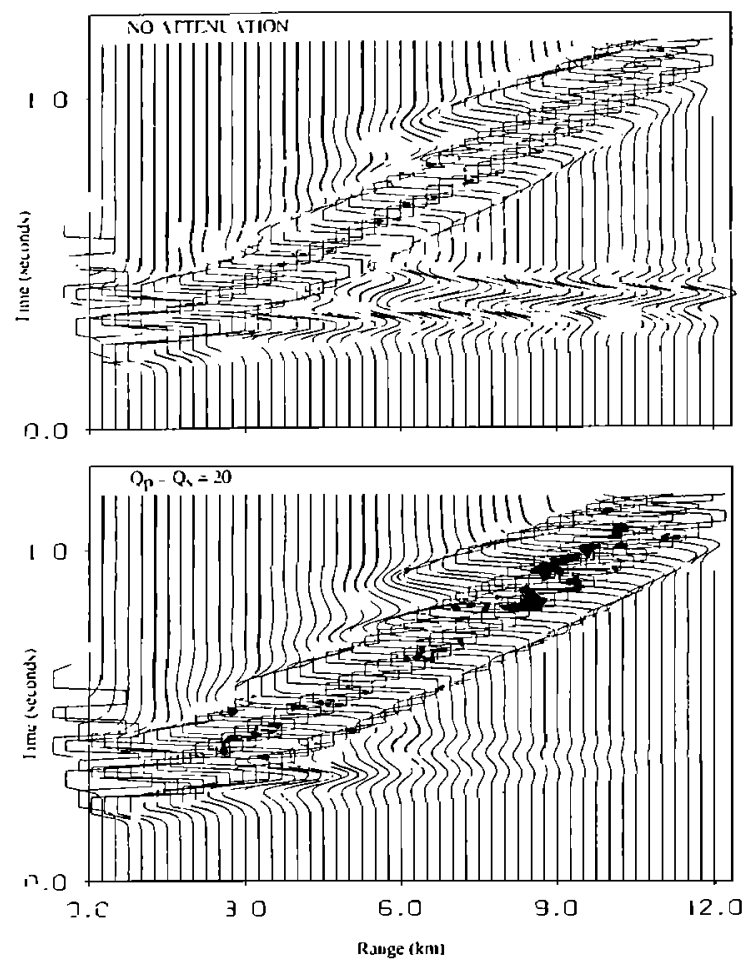

FIG. 11. Time series of the pressure response at the seafloor are shown for the models with and without attenuation (bottom and top, respectively). (These are the same models as in Figs. 9 and 10.) The high amplitude water waves (a combination of direct and reflected waves) are essentially identical in the two models except near the critical point. The compressional head wave amplitude is dramatically reduced by the attenuation as expected. The record sections have been reduced at $1.7 \mathrm{k} / \mathrm{s}$.

independent $Q$ " mean in the presence of strong gradients and scattering such as observed at the seafloor? Are the assumptions made in measuring $Q$ (for example by the spectral ratio method) valid for sound crossing rough boundaries and traveling through media with volume heterogeneities and velocity gradients?

\section{ACKNOWLEDGMENTS}

This work was carried out under Office of Naval Research Grant no. N00014-89-J-1012. WHOI Contribution Number 8480.

Abramowitz, M., and Stegun, I. A. (1965). Handbook of Mathematical Functions (Dover, New York).

Aki, K., and Richards, P. G. (1980). Quantitative Seismology: Theory and Methods (Freeman, San Francisco), Ist ed.

Alford, R. M., Kelly, K. R., and Boore, D. M. (1974). "Accuracy of finite-difference modeling of the acoustic wave equation," Geophysics 39, 834-842.

Alterman, Z. S., and Aboudi, J. (1970). "Source of finite extent, applied force and couple in an elastic half-space," Geophys. J. R. Astron. Soc. 21, 47-64.

Clayton, R., and Engquist, B. (1977). "Absorbing boundary conditions for acoustic and elastic wave equations," Bull. Seismol. Soc. Am. 67, $1529-1540$.

Day, S. M., and Minster, J. B. (1984). "Numerical simulation of attenuated wavefields using a Padé approximant method," Geophys. J. R. Astron. Soc. 78, 105-118.

Dougherty, M. E., and Stephen, R. A. (1988). "Seismic energy partitioning and scattering in laterally heterogeneous ocean crust," Pure Appl. Geophys. 128, 195-229. 
Dougherty, M. E., and Stephen, R. A. (1991). "Seismo/acoustic propagation through rough seafloors," J. Acoust. Soc. Am. 90, 2637-2651.

Fuchs, K., and Müller, G. (1971). "Computation of synthetic seismograms with the reflectivity method and comparison with observations," Geophys. J. R. Astron. Soc. 23, 417-433.

Futterman, W. I. (1962). "Dispersive body waves," J. Geophys. Res. 67, 5279-5291.

Ganley, D. C., and Kanasewich, E. R. (1980). "Measurement of absorption and dispersion from check shot surveys," J. Geophys. Res. 85, 5219-5226.

Hamilton, E. L. (1976a). "Sound attenuation as a function of depth in the seafloor," J. Acoust. Soc. Am. 59, 528-535.

Hamilton, E. L. (1976b). "Attenuation of shear waves in marine sediments," J. Acoust. Soc. Am. 60, 334-338.

Jackson, D. D., and Anderson, D. L. (1970). "Physical mechanisms of seismic attenuation," Rev. Geophys. Space Phys. 8, 1-63.

Jacobson, R. S., and Lewis, B. T. R. (1990). "The first direct measurements of upper oceanic crustal compressional wave attenuation," $J$. Geophys. Res. 95, 17,417-17,429.
Liu, H. P., Anderson, D. L., and Kanamori, H. (1976). "Velocity dispersion due to anelasticity; implications for seismology and mantle composition," Geophys. J. R. Astron. Soc. 47, 41-58.

Nowick, A. S., and Berry, B. S. (1972). Anelastic Relaxation in Crystalline Solids (Academic, New York).

Stephen, R. A. (1983). "A comparison of finite difference and reflectivity seismograms for marine models," Geophys. J. R. Astron. Soc. 72, 3957.

Stephen, R. A. (1988). "A review of finite difference methods for seismoacoustics problems at the seafloor," Rev. Geophys. 26, 445-458.

Stephen, R. A. (1991). "Finite difference modelling of shear waves," in Shear Waves in Marine Sediments, edited by J. M. Hovem, M. D. Richardson, and R. D. Stoll (Kluwer Academic, Dordrecht), pp. 471478.

Stephen, R. A., Pardo-Casas, F., and Cheng, C. H. (1985). "Finitedifference synthetic acoustic logs," Geophysies 50, 1588-1609.

Virieux, J. (1986). "P-SV wave propagation in heterogeneous media: Velocity-stress finite-difference method," Geophysics 51, 889-901. 